\title{
Rapunzel syndrome: a rare form of trichobezoar in the stomach with some extension into the small intestine
}

\author{
Anja Blejc Novak ${ }^{1}$, Živa Zupančičc ${ }^{2}$, Domen Plut ${ }^{2}$, Diana Gvardijančič ${ }^{3}$, Matjaž Homan ${ }^{4 凶}$
}

\begin{abstract}
Trichobezoars are gastric foreign bodies rarely found in children. They are mainly composed of swallowed hair. Rapunzel syndrome is a very rare form of trichobezoar characterized by extension of the ingested hair from the stomach into the small intestine or even beyond. Clinical presentation can range from an asymptomatic abdominal mass to severe symptoms: gastric outlet obstruction, perforation of the stomach, and acute pancreatitis. Trichobezoars are usually treated with surgical removal. Here we present the case of an 8-year-old girl in whom a firm non-tender mass in the epigastric region was palpated during a regular outpatient clinic visit. The girl reported some slight abdominal discomfort and early satiety during meals. Subsequently, focal alopecia was detected. A trichobezoar in the stomach with some extension into the duodenum was diagnosed by ultrasound, and later confirmed by a barium swallow study and CT scan. The trichobezoar was successfully surgically removed.
\end{abstract}

Keywords: trichobezoar, Rapunzel syndrome, foreign body, swallowed hair, alopecia

Received: 30 December 2017 | Returned for modification: 5 February 2018 | Accepted: 12 February 2018

\section{Introduction}

Bezoars are accumulations of undigested material in the gastrointestinal tract. The word bezoar comes from Arabic bedzehr or Persian padzahr, meaning 'protecting against poisons'. In history, bezoars from animal guts were used as precious stones and antidotes to poisons. Today they are still used in traditional Chinese medicine to prevent and treat cerebral vascular spasm (1). Bezoars are classified according to their primary constituents: hair (trichobezoars), vegetable or fruit fiber (phytobezoars), persimmon fiber (diospyrobezoars), milk curd (lactobezoars), medications (farmacobezoars), and miscellaneous materials (plastic gloves, paper tissue, etc.) (2). The majority of bezoars occur in the first three decades of life, mostly in patients with an underlying psychiatric disorder (3). The most common bezoars in children are trichobezoars (4). Rapunzel syndrome is a rare form of trichobezoar defined as a trichobezoar in the stomach with some extension into the small intestine (1). The syndrome is named after a fairy tale written in 1812 by the Brothers Grimm about the young maiden Rapunzel, who lowered her long hair to the ground in her prison tower to permit a prince to climb up and rescue her (2). The syndrome was first described by Vaughan et al. in 1968, and it is an uncommon diagnosis in children, with fewer than 40 cases reported in the literature (5).

We report the case of a young girl with Rapunzel syndrome that presented with an epigastric mass and focal alopecia.

\section{Case report}

An 8-year-old girl was referred for abdominal ultrasound examination due to a palpable firm mass in the epigastrium detected upon physical examination during a regular outpatient clinic visit. On the basis of ultrasound findings of a large echogenic mass within the lumen of the stomach, a large bezoar was suspected (Fig. 1). The girl's medical history revealed that she had the bad habit of putting her long hair into her mouth for the previous 4 years (trichotillomania). Upon detailed clinical examination of the scalp, alopecia of the occipital region was determined (Fig. 2). We assumed that the girl was also swallowing her hair (trichophagia), and therefore a bezoar composed of hair was highly suspected. Baseline laboratory tests were all within normal limits. To determine the size and the extent of the trichobezoar, a barium swallow study and CT scan were performed. These studies showed the trichobezoar as a large filling defect $(20 \times 6 \mathrm{~cm})$ within the markedly distended stomach and some extension of the trichobezoar into the duodenum, characteristic of Rapunzel syndrome (Figs. $3,4)$. Upper endoscopy was not necessary because of the clear diagnosis. The girl underwent abdominal surgery. Gastrotomy was performed and a large brownish trichobezoar in the shape of the stomach was found and extracted as a whole (Figs. 5a, 5b). Postoperatively, the patient made an uneventful recovery. She was discharged from the hospital after five days. Psychiatric follow-up was arranged to prevent recurrence of the disease.

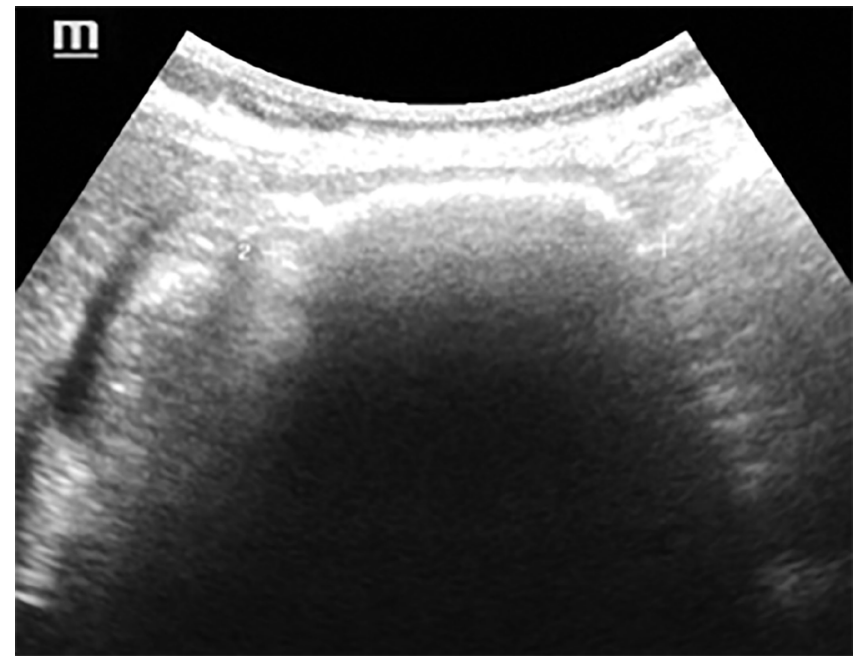

Figure 1 | Ultrasound shows a large echogenic mass in the lumen of the stomach. 


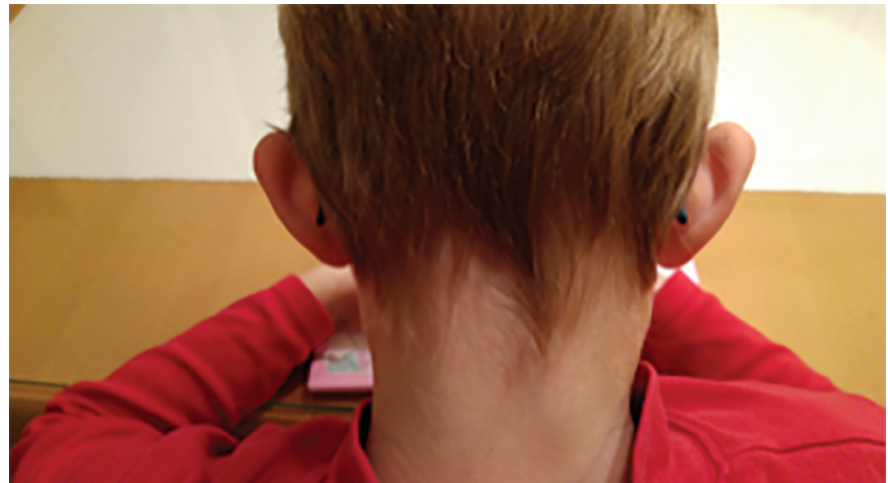

Figure 2 | Alopecia in the occipital region.

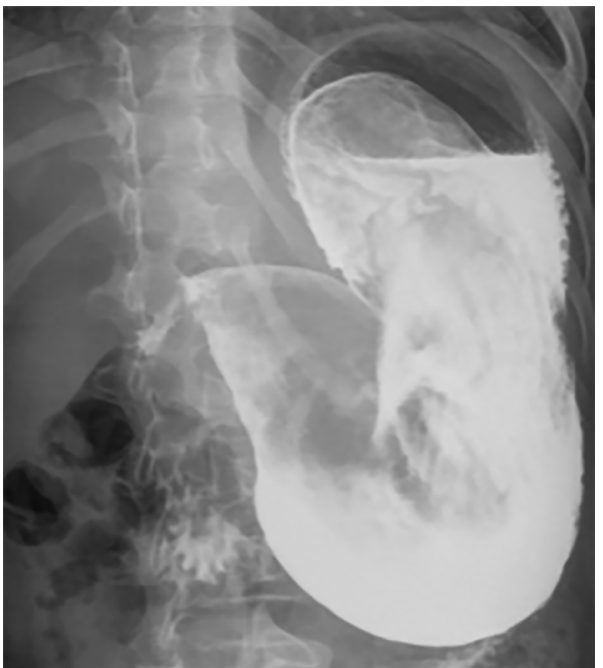

Figure 3 | A barium swallow study shows a large filling defect in the stomach.

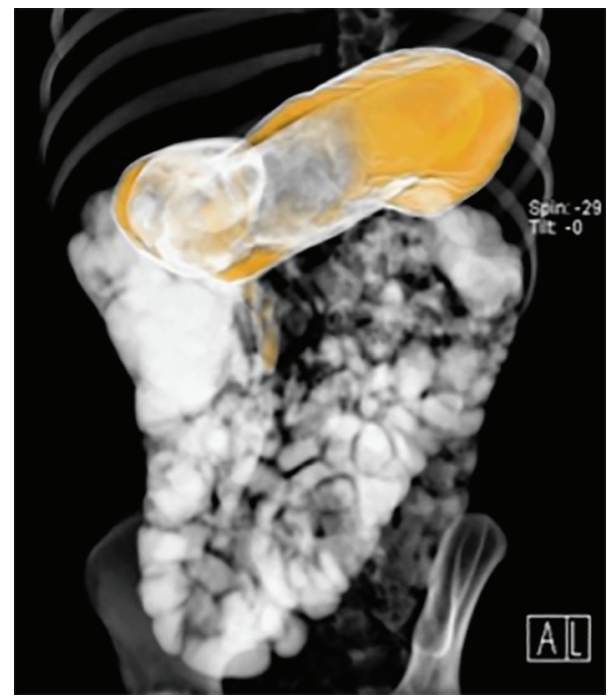

Figure 4 | CT scan (color reconstruction) shows the trichobezoar in the stomach with some extension into the duodenum (Rapunzel syndrome).

\section{Discussion}

Trichotillomania usually results in alopecia, as in our case. It manifests with irregular areas of hair loss and with no clear area of baldness (Fig. 2). Common causes of hair loss in children include telogen effluvium, tinea capitis, bacterial infections, traction alopecia, trichotillomania, and alopecia areata. Other less common causes of alopecia are thyroid disorders, illnesses such as systemic lupus erythematosus, diabetes mellitus, or iron-deficiency anemia, malnutrition, and structural abnormalities of the hair shaft $(6,7,8)$. The word trichotillomania is derived from Greek
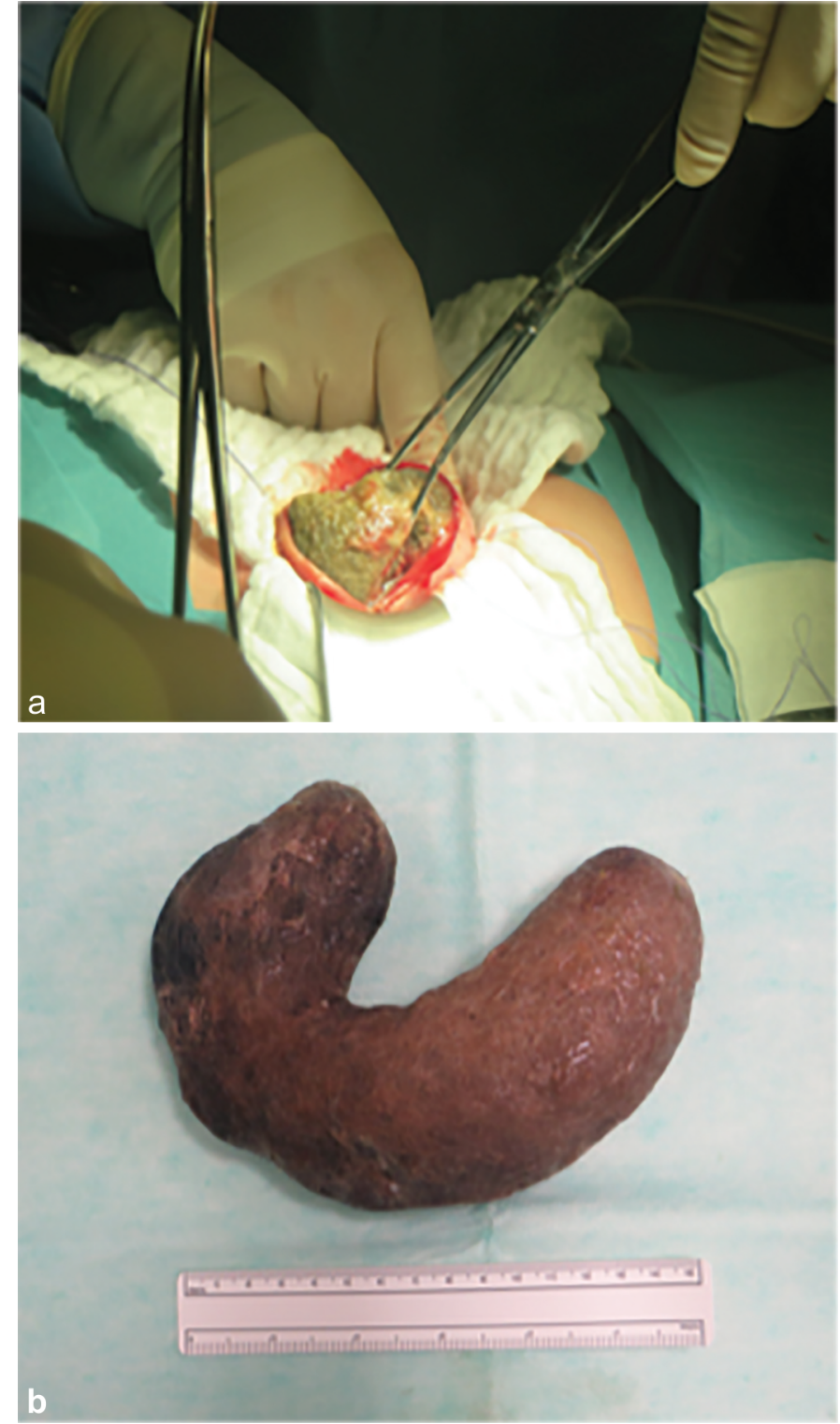

Figure 5| a) Bezoar removal, b) $20 \mathrm{~cm}$ trichobezoar.

thrix 'hair', tillein 'to pull', and mania 'madness'. The prevalence among children and adolescents has been estimated at less than $1 \%$ (9).

Most patients with trichobezoars suffer from psychiatric disorders including trichotillomania and trichophagia, as in our case. Among those that suffer from trichotillomania, only 30\% will engage in trichophagia, and of the latter only $1 \%$ develop a trichobezoar (2). Some other psychiatric disorders may also be associated with trichobezoar: mental disorders, abuse, pica, obsessive-compulsive disorder, depression, and anorexia nervosa (6).

Hair is retained in the stomach because it escapes peristaltic activity due to its smooth and slippery surface. The hair eventually becomes enmeshed into a ball, which finally assumes the shape of the stomach. Trichobezoars are brownish in color due to protein denaturation by acid, glistening from retained mucus, and foul smelling due to the decomposition of fat residues within the hair, which also results in halitosis (2). These features were also present in our case (Figs. 3, 4, 5a, 5b).

Clinical presentation mainly depends on the size of trichobezoar. Small gastric bezoars are usually asymptomatic. As bezoars enlarge with time, a variety of symptoms develop, including abdominal discomfort and pain, early satiety, postprandial fullness, nausea, and vomiting. Iron-deficiency anemia can also occur. In 
our case, the bezoar was large; however, the girl reported only early satiety and minor abdominal pain. Large bezoars can cause serious complications, such as gastric outlet obstruction, obstructive jaundice, gastrointestinal bleeding, protein-losing enteropathy, acute pancreatitis, small-intestine obstruction, perforation, peritonitis, and intussusceptions. Therefore, recognition and timely treatment of the bezoar are of major importance (10).

Diagnostic imaging studies are usually performed in patients with nonspecific upper gastrointestinal symptoms and in patients with possible complications. With imaging studies trichobezoars are detectable as mass lesions within the stomach, as was also the case in our patient (Figs. 1, 3, 4). In Rapunzel syndrome, CT is important for estimating the extension of the trichobezoar into the small intestine.

Upper gastrointestinal endoscopy is usually performed to confirm the diagnosis and to reveal the constituents of the bezoar. In some types of bezoars, such as phytobezoars and lactobezoars, endoscopy can also be an effective therapeutic option to eliminate the bezoar (1). In our patient, endoscopy was not performed because a clear diagnosis of the size and extension of the bezoar was achieved using imaging studies. Moreover, focal alopecia was discovered, and therefore a trichobezoar was expected. It is known that trichobezoars cannot be removed though an endoscopic procedure due to their size and consistency (11).

Treatment is essentially surgical. Laparotomy with extraction of the bezoar is done with exploration of rest of the small intestine to look for detached bezoars. Trichobezoar extensions are removed and small intestine segments that show extensive ulcerations or gangrene are resected. In our patient, surgeons performed a gastrotomy and the trichobezoar mass was removed in one piece. At the time of surgery there was no evidence of trichobezoar extension into the duodenum and no larger detached parts of bezoar distally within the intestine. The extension of the trichobezoar in the duodenum diagnosed by CT was probably detached and eliminated before surgery. Laparoscopic removal of a trichobezoar could be a surgical option; however, it may be hard to achieve when trichobezoars are large. In addition, there is a lack of surgical experience with this technique because of the rarity of trichobezoars, especially the entity of Rapunzel syndrome $(6,12)$. Postoperatively, the girl made an uneventful recovery. Already during her stay at the surgical ward the girl had her long hair cut short to prevent further swallowing of long hair.

Psychiatric evaluation, treatment, and parental counseling, as well as regular follow-ups, are an important part of the treatment to prevent trichophagia and the recurrence of a trichobezoar (2). Our patient was referred for psychiatric evaluation and counseling.

\section{Conclusion}

A trichobezoar should be considered in young female patients with focal or generalized alopecia. It should also be considered in patients with non-specific or severe abdominal symptoms in association with alopecia. Rapunzel syndrome is a rare variant of trichobezoar in the stomach extending from the stomach into the small intestine. Surgical removal is the optimal method of treatment. Many patients have psychiatric pathology with emotional problems, family discord, and a history of neglect, abuse, or mental retardation. Proper psychiatric counseling and treatment are needed.

\section{References}

1. Gonuguntla V, Joshi DD. Rapunzel syndrome: a comprehensive review of an unusual case of trichobezoar. Clin Med Res. 2009;7:99-102.

2. Bashir EA, Samiullah, Sadiq MA, Yusuf O, Karim K. Rapunzel Syndrom. J Ayub Med Coll Abbottabad. 2010;22:218-20.

3. Cardoso Della Bidia F, Bastos Guimaraes De Almeida P, Pinto De Moraes H, Filqueiras M, Zorron R. Laparoscopic treatment of gastric trichobezoar. Bras J Video-Sur. 2008;1:178-81.

4. Sanders MK. Bezoars: From mystical charms to medical and nutritional management. Practical Gastroenterol. [Online]. [cited 2017 Jan 10]. Available from: https://med.virginia.edu/ginutrition/wp-content/uploads/sites/199/2015/11/ Sanders-Jan-04.pdf.

5. Vaughan ED, Jr, Sawyers JL, Scott HW Jr. The Rapunzel syndrome. An unusual complication of intestinal bezoar. Surgery. 1968;63:339-43.

6. Gorter RR, Kneepkens CM, Mattens EC, Aronson DC, Heij HA. Management of trichobezoar: case report and literature review. Pediatr Surg Int. 2010;26:45763.

7. Mandt N, Vogt A, Blume-Peytavi U. Differential diagnosis of hair loss in children. J Dtsch Dermatol Ges. 2004;2:399-411.

8. Castelo-Soccio LA. Hair manifestations of systemic disease. Curr Probl Pediatr Adolesc Health Care. 2012;42:198-203.

9. Zimova J, Zimova P. Trichotillomania: bizzare patern of hair loss at 11-year-old girl. Acta Dermatovenerologica Croat. 2016;24:150-3.

10. Sharma P, Kumar S, Kumar B, Jadon RS. Rapunzel syndrome: a rare cause of vomiting and pain abdomen. JK Science. 2012;14:146-8.

11. Kaushik NK, Sharma YP, Negi A, Jaswal A. Image-gastric trichobezoar. Indian J Radiol Imaging. 1999;9:137-9.

12. Jatal SN, Jamadar NP, Jadhav B, Siddiqui S, Ingle SB. Extremely unusual case of gastrointestinal trichobezoar. World J Clin Cases. 2015;3:466-9. 